\title{
Tuberculosis adrenal en un paciente inmunocompetente: reporte de un caso
}

\author{
María Alejandra Pérez ${ }^{1}$, Julián Naranjo Millán ${ }^{1}$, Henry Augusto Millán ${ }^{2}$, \\ Freddy Andrés Luna Vela², Andrés Flórez ${ }^{3}$
}

\begin{abstract}
${ }^{1}$ Médico residente de medicina interna, Universidad Nuestra Señora del Rosario, Fundación Cardioinfantil Instituto de Cardiología, Bogotá, Colombia.

${ }^{2}$ Médico Internista Fundación Cardioinfantil Instituto de Cardiología, Bogotá, Colombia.

${ }^{3}$ Médico Internista, Fellow endocrinología. Fundación Universitaria de Ciencias de la salud - Hospital de San José, Bogotá, Colombia.
\end{abstract}

Autor de correspondencia: María Alejandra Pérez Ardila, correo electrónico: mariaperezardila@gmail.com

Aporte de los autores: La recolección de los datos de la historia clínica estuvo a cargo de María Alejandra Pérez y Julián Naranjo. La recolección de la bibliografía usada para la revisión de tema contó con la participación de todos los autores. La redacción de la epidemiología, manifestaciones clínicas y de laboratorio a cargo de Julián Naranjo y Freddy Luna, y la redacción de mecanismos fisiopatológicos, criterios diagnósticos y tratamiento a cargo de María Alejandra Pérez y Andrés Flórez.

Fecha de aceptación: 28/06/2018

\section{Resumen}

$\mathrm{E}$ n la primera mitad del siglo XX, la infección por tuberculosis era la primera causa de insuficiencia suprarrenal, ahora desplazada por la insuficiencia suprarrenal autoinmune primaria; sin embargo, en países en desarrollo la adrenalitis por tuberculosis sigue siendo una de las etiologías más frecuentes ${ }^{(1)}$. Presentamos el caso de un hombre de 53 años con cambios de hiperpigmentación generalizada, astenia y adinamia previo al inicio de los síntomas respiratorios. Se realizó el diagnóstico de tuberculosis pulmonar y durante el tratamiento con isoniacida, rifampicina, etambutol y pirazinamida presentó crisis suprarrenal.

Palabras clave: Mycobacterium tuberculosis, tuberculosis, insuficiencia adrenal, enfermedad de Addison.

\section{Abstract}

In the first half of the 20th century Tuberculosis infection was the first cause of adrenal insufficiency, now displaced by primary autoimmune insufficiency; however, in developing countries Tuberculosis Adrenalitis remains one of the etiologies to be considered. We present the case of a 53-year-old man with changes in hyperpigmentation, asthenia and adynamia prior to respiratory symptoms, initially diagnosed pulmonary tuberculosis and under treatment with Isoniazid, Rifampicin, Ethambutol and Pirazinamide presented signs of adrenal crisis.

Keywords: Mycobacterium tuberculosis, Tuberculosis, Adrenal Insufficiency.

\section{Consideraciones éticas}

El caso que se presenta corresponde a un paciente masculino adulto atendido en la Fundación Cardioinfantil Instituto de Cardiología; la publicación del mismo contó con el consentimiento previo del paciente, se explicó previamente el propósito del reporte, siendo voluntaria su participación en el mismo; durante el proceso se garantizó la confidencialidad de la información y no se expuso a tratamientos experimentales.

\section{Introducción}

La primera descripción del bacilo Mycobacterium tuberculosis se realizó en Berlín por Robert Koch, lo que lo hizo merecedor del premio Nobel en 1905. En Colombia se registró la mayor epidemia por tuberculosis pulmonar entre 1875 y $1914^{(2)}$. Favoreciendo la creación de pabellones para pacientes con tuberculosis. Hasta el día de hoy se han implementado varias estrategias para el control de la infección en el país; sin embargo, para el 2014 se notificaron 12.824 casos anuales, de los cuales $19,6 \%$ correspondían a manifestaciones de tipo extrapulmonar ${ }^{(3)}$. Hasta la primera mitad del siglo XX, la infección por tuberculosis fue la primera causa de insuficiencia suprarrenal, actualmente la etiología autoinmune es la causa más frecuente ${ }^{(4,5)}$; sin embargo, en India se describió que la infección por tuberculosis se encontró como etiología en $47 \%$ de los $\operatorname{casos}^{(1)}$, lo que debe alertar de esta etiología en pases en desarrollo como el nuestro. $\mathrm{Ni}$ en Latinoamérica ni en nuestro países existe un registro que permita establecer la prevalencia de insuficiencia suprarrenal en la infección por Mycobacterium tuberculosis.

\section{Caso clínico}

Paciente de 53 años quien reside en Bogotá, con diagnóstico de tuberculosis pulmonar un mes antes de la consulta, en 
tratamiento con isoniacida, rifampicina, pirazinamida y etambutol, se realizó serología para VIH que resultó negativa. Consultó por empeoramiento de la disnea, astenia y escalofríos, esta sintomatología coincidió con el inicio de la terapia. Refería además cambios en la coloración en la piel de 18 meses de evolución.

$\mathrm{Al}$ ingreso se encontró hipotenso, sin taquicardia; con hiperpigmentación generalizada, sin otros hallazgos anormales al examen físico. En los laboratorios se reportó hiponatremia e hipercaliemia, con glucosa sérica normal, en los gases arteriales se encontró acidosis metabólica compensada (tabla 1). Por lo anterior, se sospechó insuficiencia suprarrenal, se tomó muestra para cortisol y se inició manejo con hidrocortisona, hidratación intravenosa y se solicitó tomografía (TC) de tórax y de abdomen con protocolo para glándulas suprarrenales.

En la tomografía de tórax se observó compromiso micronodular de predominio centrolobulillar y una zona de consolidación en el lóbulo medio, por lo que fue llevado a fibrobroncoscopia con aislamiento de Mycobacterium tuberculosis en el cultivo del lavado broncoalveolar. En la TC de abdomen con protocolo para glándulas suprarrenales no se observaron lesiones (figura 1).

Se realizó el diagnóstico de insuficiencia suprarrenal teniendo en cuenta el resultado de cortisol sérico en $2,5 \mathrm{mcg} / \mathrm{dL}$ ( $<10 \mathrm{mcg} / \mathrm{dL}$ se considera bajo para paciente crítico). La ACTH tomada después de obtener el resultado del cortisol fue normal, el valor esperado dado que fue tomada 48 horas después de iniciar la hidrocortisona (tabla 1). Se realizó descenso progresivo de la dosis de hidrocortisona intravenosa hasta $50 \mathrm{mg}$ al día, en este momento se realizó cambio a hidrocortisona vía oral a dosis de $20 \mathrm{mg}$ al día (10 mg a las 8 a. m. y $10 \mathrm{mg}$ a las 3 p. m.), con control de los síntomas, estabilidad hemodinámica y normalización de los electrolitos. Se indició continuar esta misma dosis de forma ambulatoria.

Figura 1. Tomografía de abdomen contrastado.

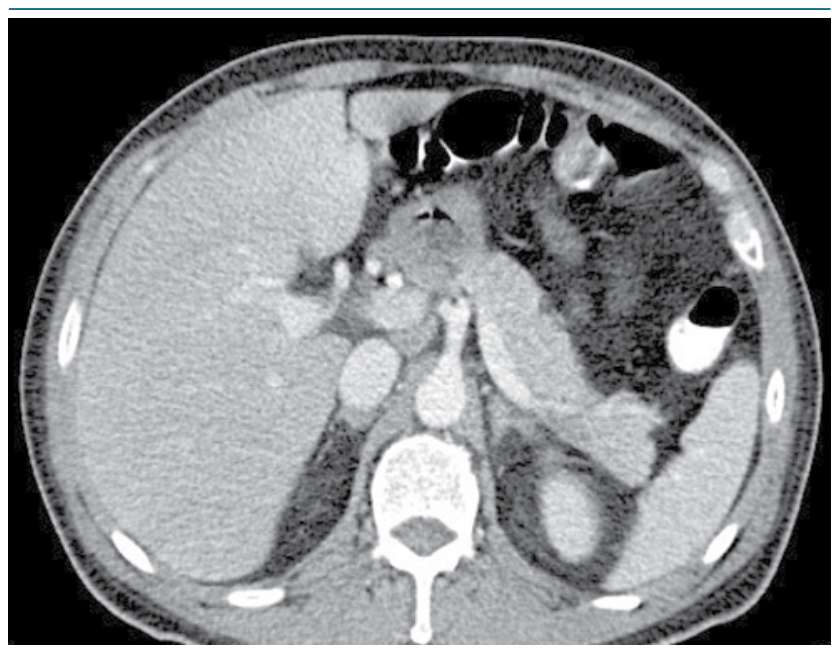

Glándulas suprarrenales de tamaño normal, sin observarse alteraciones en su densidad o masas.
Tabla 1. Laboratorios iniciales del paciente

\begin{tabular}{|c|c|c|}
\hline Laboratorios & $\begin{array}{l}\text { Valor del } \\
\text { paciente }\end{array}$ & Valor normal \\
\hline Leucocitos & $4840 \mathrm{cel} / \mathrm{mcl}$ & $5000-10000 \mathrm{cel} / \mathrm{mcl}$ \\
\hline Granulocitos & $2680 \mathrm{cel} / \mathrm{mcl}$ & $2000-7000 \mathrm{cel} / \mathrm{mcl}$ \\
\hline Linfocitos & $1510 \mathrm{cel} / \mathrm{mcl}$ & $1500-4000 \mathrm{cel} / \mathrm{mcl}$ \\
\hline Eosinófilos & $40 \mathrm{cel} / \mathrm{mcl}$ & $0-400 \mathrm{cel} / \mathrm{mcl}$ \\
\hline Monocitos & $550 \mathrm{cel} / \mathrm{mcl}$ & $200-800 \mathrm{cel} / \mathrm{mcl}$ \\
\hline Hemoglobina & $13,6 \mathrm{~g} / \mathrm{dL} \mathrm{g}$ & $13,5-18 \mathrm{gr} / \mathrm{dL}$ \\
\hline Hematocrito & $40,3 \%$ & $40 \%-54 \%$ \\
\hline Plaquetas & $552000 \mathrm{cel} / \mathrm{mcl}$ & $150000-450000 \mathrm{cel} / \mathrm{mcl}$ \\
\hline Sodio & $118 \mathrm{meq} / \mathrm{L}$ & $136-142 \mathrm{mEq} / \mathrm{L}$ \\
\hline Potasio & $7,5 \mathrm{meq} / \mathrm{L}$ & $3,5-5,5 \mathrm{mEq} / \mathrm{L}$ \\
\hline Creatinina & $2,1 \mathrm{mg} / \mathrm{dL}$ & $0,7-1,3 \mathrm{mg} / \mathrm{dL}$ \\
\hline BUN & $37 \mathrm{mg} / \mathrm{dL}$ & $8,4-25 \mathrm{mg} / \mathrm{dL}$ \\
\hline Glicemia & $109 \mathrm{mg} / \mathrm{dL}$ & $70-100 \mathrm{mg} / \mathrm{dL}$ \\
\hline $\mathrm{PH}$ & 7,44 & $7,39-7,47$ \\
\hline $\mathrm{PCO} 2$ & $23,8 \mathrm{mmHg}$ & $28,3-38,7 \mathrm{mmHg}$ \\
\hline $\mathrm{PO} 2$ & $69 \mathrm{mmHg}$ & $63,3 \mathrm{mmHg}$ \\
\hline $\mathrm{HCO} 3$ & $15,8 \mathrm{mmol} / \mathrm{L}$ & $19,1-24,7 \mathrm{mmol} / \mathrm{L}$ \\
\hline Lactato & $1,56 \mathrm{mmol} / \mathrm{L}$ & $0,4-2,2 \mathrm{mmol} / \mathrm{L}$ \\
\hline $\mathrm{VIH}$ & Negativo & \\
\hline Cortisol AM & $2,50 \mathrm{ug} / \mathrm{dL}$ & $3,7 \mathrm{ug} / \mathrm{dL}-19,4 \mathrm{ug} / \mathrm{dL}$ \\
\hline ACTH & $13,1 \mathrm{pg} / \mathrm{mL}$ & $7,2-63,3 \mathrm{pg} / \mathrm{uL}$ \\
\hline
\end{tabular}

Cel: células, mcl: microlitro, g: gramos, dL: decilitro, meq: miliequivalentes, mmHg: milímetros de mercurio, mmol: milimoles, L: litro, pg: picogramos.

\section{Discusión}

La infección por tuberculosis puede tener cualquier localización anatómica, pudiendo afectar las glándulas suprarrenales ${ }^{(6)}$. La relación entre estas dos condiciones había sido reportada por el Dr. Thomas Addison en su descripción de 11 casos de insuficiencia suprarrenal en infección por Mycobacterium tuberculosis ${ }^{(7)}$.

Sin embargo, actualmente en los países desarrollados la etiología infecciosa de la insuficiencia suprarrenal es del $10 \%{ }^{(8)}$, siendo la infección por M. tuberculosis el 1\% de las causas. Otros microorganismos que pueden causar infección adrenal son: criptococo, histoplasma, coccidioides, paracoccidioides, VIH, citomegalovirus, echinococcus sp. y trypanozoma sp..$^{(5,8)}$.

Se han propuesto varios mecanismos por los que la infección por M. tuberculosis genera insuficiencia suprarrenal, los cuales incluyen:

- La rifampicina puede inducir insuficiencia suprarrenal al acelerar el metabolismo del $\operatorname{cortisol}^{(9)}$. Es un inductor de citocromo P-450 que puede aumentar el metabolismo de hormonas como el cortisol; esto debido a la actividad 6B-hidroxilasa del citocromo $^{(10,11)}$. Sin embargo, dada su alta efectividad para el tratamiento de la tuberculosis, se consideró continuarla a pesar de la insuficiencia suprarrenal del paciente. 
- En la infección por tuberculosis hay activación del eje hipotálamo-hipófisis-adrenal, aumentando la secreción de cortisol, que resulta en una mayor producción de linfocitos TH2, esta disfunción en los linfocitos T puede llevar a daño mediado inmunológicamente en los tejidos ${ }^{(12)}$.

- Se ha observado que la liberación de citocinas como IL-1, IL-6 y TNF (Factor de necrosis tumoral) durante la infección por Mycobacterium tuberculosis, lleva a liberación del factor de corticotropina y activación del eje hipotálamo-hipófisisadrenal $^{(12,13,14)}$, generando también daño directo sobre la corteza adrenal.

Los estudios realizados al momento para establecer el perfil hormonal en pacientes con tuberculosis pulmonar son observacionales y han mostrado resultados discordantes, por lo que aún no se puede establecer la relación entre esta infección y los niveles de cortisol.

En el estudio de Bongiovanni et al. en 12 pacientes con tuberculosis pulmonar se encontraron niveles elevados de estas citocinas, PCR, cortisol y aumento de la relación cortisol/DHEA (dehidroepiandrosterona), con concentraciones disminuidas de DHEA cuando se compara con controles sanos ${ }^{(15)}$. Sin embargo, en el estudio de Baker et al. no se encontraron diferencias en el cortisol en orina de 24 horas, cortisol salivar, cortisol pos dexametasona o la respuesta adrenal a ACTH. Se encontró un aumento de la relación cortisol/cortisona en lavado broncoalveolar pero no a nivel plasmático ${ }^{(16)}$.

La relación entre tuberculosis pulmonar e insuficiencia adrenal fue confirmada en el estudio realizado por Laway et al. ${ }^{(17)}$, en el que se analizaron 45 pacientes con tuberculosis pulmonar activa y 45 controles sanos. Se encontró que el cortisol basal y pos estímulo con ACTH eran más bajos en los casos. Tras terminar el tratamiento se realizó una nueva medición, la cual mostró que el cortisol basal no tuvo cambios significativos. En este estudio también se analizaron hallazgos tomográficos de las glándulas suprarrenales, encontrando que los pacientes con tuberculosis tenían mayor tamaño y grosor de las mismas ${ }^{(17)}$.

Guo et al. ${ }^{(18)}$ reportaron las imágenes de 42 pacientes con tuberculosis adrenal, describiendo que 91\% tenían aumento suprarrenal bilateral y 7\% unilateral. Algunos hallazgos como calcificaciones eran más frecuentes a medida que la enfermedad progresaba. La calcificación se relacionó con formación de granulomas encapsulados que favorecen la precipitación de calcio; sin embargo, no es un hallazgo exclusivo de infección por tuberculosis, en la histoplasmosis, hemorragia suprarrenal y blastomicosis también se producen depósitos de calcio. En esta misma serie, el $2 \%$ de los pacientes presentaron suprarrenales de tamaño normal, como lo descrito en este caso ${ }^{(18)}$.

Por lo tanto, en los pacientes con tuberculosis pulmonar se debe prestar atención a los síntomas que sugieren compromiso adrenal, los cuales pueden ser inespecíficos, como fatiga crónica, anorexia, pérdida no intencional de peso, náuseas, vómito y dolor abdominal, que pueden asociarse a compromiso pulmo- nar o al tratamiento; otros hallazgos más específicos como hipotensión e hipoglucemia pueden presentarse en el contexto de una crisis suprarrenal ${ }^{(19)}$. Otros signos clínicos de insuficiencia suprarrenal primaria son: hiponatremia e hipocaliemia: secundarias a hipoaldosteronismo, e hiperpigmentación de la piel: secundaria al aumento en la producción de propiomelanocortina, con la producción concomitante de hormona estimulante de melanocitos.

Niveles de cortisol en la mañana (8 a 9 a. m.) menores a 5 $\mathrm{mcg} / \mathrm{dL}$ (140 nmol/L) en combinación con niveles de ACTH, que pueden tomarse durante el test de estímulo con corticotropina o de forma simultánea con la prueba de cortisol y que estén dos veces sobre el límite superior, son sugestivos de insuficiencia suprarrenal primaria ${ }^{(20)}$.

La confirmación del diagnóstico se realiza mediante estimulación con corticotropina intravenosa ( $250 \mathrm{mcg}$ ), con niveles de cortisol menores a $18 \mathrm{mcg} / \mathrm{dL}$ (500 nmol/L) a los 30 o $60 \mathrm{mi}$ nutos se confirma el diagnóstico. Sin embargo, no es necesario realizar esta prueba si retrasa la intervención en pacientes con inestabilidad hemodinámica y un cuadro clínico sugestivo de insuficiencia suprarrenal, como el presentado en nuestro caso ${ }^{(20)}$.

En los pacientes críticos no existe consenso sobre el límite de cortisol para el diagnóstico, esto teniendo en cuenta que los pacientes críticamente enfermos tienen niveles más altos de cortisol basal; además, más del 90\% del cortisol circulante se encuentra unido a proteínas, por lo que la presencia de hipoalbuminemia en este grupo de pacientes puede generar que el cortisol total sea un indicador no confiable ${ }^{(21)}$. Se ha recomendado un límite de $10 \mathrm{mcg} / \mathrm{dL}$ como límite para iniciar manejo con glucocorticoides en los pacientes críticamente enfermos con hipoproteinemia significativa ${ }^{(21)}$. En los pacientes en quienes se obtenga un valor de cortisol aleatorio en el límite inferior se recomienda realizar un test de estimulación con corticotropina, los niveles esperados varían en los pacientes críticamente enfermos, de esta forma en quienes tengan albúmina > $2,5 \mathrm{~g} / \mathrm{dL}$ se espera un valor de cortisol $>22 \mathrm{mcg} / \mathrm{dL}$, en hipoalbuminemia el límite de valor de cortisol es de $15 \mathrm{mcg} / \mathrm{dL}^{(21)}$.

La hidrocortisona es el corticoide de elección, por su efecto glucocorticoide y mineralocorticoide, menor riesgo de hiperglucemia y amplio rango terapéutico, la dosis de inicio es de 15 a $25 \mathrm{mg}$ dividido en dos a tres dosis al día; la mayor dosis se administra en la mañana; la siguiente dosis 2 horas después del almuerzo, si se utilizan tres dosis, debe administrarse la última dosis 4 a 6 horas antes de dormir. En caso de no tener disponibilidad de hidrocortisona oral, una alternativa es prednisolona de 3 a $5 \mathrm{mg} / \mathrm{dL}$ de una a dos veces al día ${ }^{(20)}$.

El remplazo de mineralocorticoide se recomienda en los pacientes en quienes se confirma la deficiencia de aldosterona; se usa fludrocortisona 0,05 a 0,1 mg día, como dosis inicial. La monitorización se realiza con datos clínicos y paraclínicos, como la necesidad de sal percibida por el paciente, hipotensión postural, hipercaliemia e hiponatremia que sugieren déficit de 
mineracolocorticoides. En caso de edema, hipertensión arterial, hipocaliemia e hipernatremia se debe disminuir la dosis de mineracolocorticoides. Niveles de renina plasmática en rango superior normal son útiles para evitar uso excesivo de mineralocorticoides. La fludrocorticona debe usarse en caso de utilizar prednisolona, no es necesaria si se usa hidrocortisona, razón por la cual no se indicó de forma ambulatoria en este caso ${ }^{(20)}$.

Al comparar nuestro caso con otros casos reportados se encontró similitud en el diagnóstico previo de infección pulmonar o pleural ${ }^{(8,10)}$; sin embargo, hay otros casos con diagnóstico de insuficiencia suprarrenal durante infección diseminada ${ }^{(22,23)}$, de forma aislada ${ }^{(24,25,26)}$ o antes del diagnóstico de tuberculosis pleural o pulmonar ${ }^{(27)}$ lo que demuestra la variedad del espectro clínico de la infección por Mycobacterium tuberculosis.

El diagnóstico de insuficiencia suprarrenal primaria en nuestro caso, se realizó teniendo en cuenta los hallazgos clínicos y paraclínicos. Si bien la ACTH se encontraba en rangos normales, este resultado era esperable, dado que fue realizada durante la suplencia con hidrocortisona. Se consideró de origen primario dadas las manifestaciones de hipoaldosteronis- mo (poco frecuente en insuficiencia suprarrenal secundaria), hiperpigmentación cutánea (relacionada con aumento $\alpha \mathrm{MSH}$ [hormona estimulante de melanocitos], producto de degradación de la POMC [pro-opiomelanocortina]) y teniendo en cuenta la asociación de tuberculosis con compromiso adrenal.

A la luz de la evidencia clínica surgen algunos interrogantes, tales como: ¿Cuál es la verdadera incidencia de infección por tuberculosis adrenal en Colombia? y ¿Qué implicación en el pronóstico de los pacientes con infección por M. tuberculosis el nivel de cortisol y DHEA detectado previo al tratamiento?, esto último teniendo en cuenta la relación entre estas hormonas y la respuesta celular frente la infección.

\section{Conflicto de interés}

Los autores declaramos que no hemos recibido directa o indirectamente recursos financieros de la institución para la cual trabajamos o de un tercero para la publicación de este caso o la investigación realizada respecto al tema central. No tenemos actividades o relaciones con terceros que hayan influido en la publicación de este artículo, ni en las conclusiones presentadas.

\section{Referencias}

1. Agarwal G, Bhatia E, Pandey R, Jain SK. Clinical profile and prognosis of Addison's disease in India. Natl Med J India. 2001;14(1):23-5.

2. Ordóñez Sánchez SA, López Osma FA. Tuberculosis en Colombia, de la historia al entendimiento de la enfermedad. Tuberc Colomb from Hist to Underst Dis [Internet]. 2013;16(3):127-42.

3. INS. Protocolo De Vigilancia En Salud Publica Tuberculosis. Inst Nac Salud [Internet]. 2016;42. Available from: http://www.ins.gov.co:81/lineas-deaccion/Subdireccion-Vigilancia/sivigila/Protocolos SIVIGILA/PRO Tuberculosis.pdf

4. Bensing S, Hulting A, Husebye ES, Kämpe O, Løvås K. Epidemiology , Quality of Life and Complications of Primary Adrenal Insufficiency : a review . 2016;(April):1-27.

5. Charmandari E, Nicolaides NC, Chrousos GP. Adrenal insufficiency. Lancet. 2014;383(9935):2152-67.

6. Shah M, Reed C. Complications of tuberculosis. Curr Opin Infect Dis [Internet]. 2014;27(5):403-10. Available from: http://www.ncbi.nlm.nih.gov/ pubmed/25028786

7. Pearce JMS. Thomas Addison(1793-1860). J R Soc Med. 2004 Jun;97(6):297300.

8. Upadhyay J, Sudhindra P, Abraham G, Trivedi N. Tuberculosis of the adrenal gland: A case report and review of the literature of infections of the adrenal gland. Int J Endocrinol. 2014;2014.

9. Ray A, Suri JC, Gupta M. Case Report Rifampicin induced adrenal crisis in an uncommon setting. 2013;30(4):1-2.

10. Denny N, Raghunath S, Bhatia P, Abdelaziz M. Rifampicin-induced adrenal crisis in a patient with tuberculosis : a therapeutic challenge. 2016;

11. Wilkins EG, Hnizdo E, Cope A. Addisonian crisis induced by treatment with rifampicin. Tubercle. 1989 Mar;70(1):69-73.

12. Kelestimur $\mathrm{F}$. The endocrinology of adrenal tuberculosis: the effects of tuberculosis on the hypothalamo-pituitary-adrenal axis and adrenocortical function. J Endocrinol Invest. 2004 Apr;27(4):380-6.

13. Fukata J, Imura H, Nakao K. Cytokines as mediators in the regulation of the hypothalamic-pituitary-adrenocortical function. J Endocrinol Invest. 1994 Feb;17(2):141-55

14. Pozzoli G, Costa A, Grimaldi M, Schettini G, Preziosi P, Grossman A, et al. Lipopolysaccharide modulation of eicosanoid and corticotrophin-releasing hormone release from rat hypothalamic explants and astrocyte cultures in vitro: evidence for the involvement of prostaglandin E2 but not prostaglandin F2 alpha and lack of effect of nerve growth factor. J Endocrinol. 1994 Jan;140(1):103-9.
15. Bongiovanni B, Ariana D, Attilio LD, Santucci N, Griselda D, Lioi S, et al. Changes in the immune and endocrine responses of patients with pulmonary tuberculosis undergoing specific treatment. 2012;1262:10-5.

16. Baker RW, Walker BR, Shaw RJ, Honour JW, Jessop DS, Lightman SL, et al Increased Cortisol Cortisone Ratio in Acute Pulmonary Tuberculosis. 2000

17. Laway BA, Khan I, Shah BA, Choh NA, Bhat MA, Shah ZA. Pattern of adrenal morphology and function in pulmonary tuberculosis: Response to treatment with antitubercular therapy. Clin Endocrinol (Oxf). 2013;79(3):321-5.

18. Guo YK, Yang ZG, Li Y, Ma E Sen, Deng YP, Min PQ et al. Addison's disease due to adrenal tuberculosis: Contrast-enhanced CT features and clinical duration correlation. Eur J Radiol. 2007;62(1):126-31.

19. Chakera AJ, Vaidya B. Addison Disease in Adults: Diagnosis and Management. Am J Med [Internet]. 2010;123(5):409-13. Available from: http:// dx.doi.org/10.1016/j.amjmed.2009.12.017

20. Bornstein SR, Allolio B, Arlt W, Barthel A, Don-wauchope A, Hammer GD, et al. Diagnosis and Treatment of Primary Adrenal Insufficiency: An Endocrine Society Clinical Practice Guideline. 2016;(January):1-26.

21. Practice E. Amir H Hamrahia, Maria Fleseriu. AACE Adrenal Scientific Committee. 2017.

22. Manso MC, Rodeia SC, Rodrigues S, Domingos R. Synchronous presentation of two rare forms of extrapulmonary tuberculosis. BMJ Case Rep. 2016 Apr;2016:10.1136/bcr-2015-212917.

23. Rajasekharan C, Ajithkumar S, Anto V, Parvathy R. Extrapulmonary disseminated tuberculosis with tuberculous adrenalitis: a stitch in time saves nine. BMJ Case Rep. 2013 May;2013.

24. Sharma M, Patil A, Somani P. Bilateral adrenal tuberculosis diagnosed by endoscopic ultrasound-guided fine-needle aspiration cytology. Endoscopy. 2016;48 Suppl 1:E246-7.

25. Ikoma A, Namai K, Saito T, Kawano T, Saito T, Kasono K, et al. Unilateral active adrenal tuberculosis featuring persistent intermittent fever. Endocr J. 2004 Oct;51(5):463-6.

26. Imisairi $\mathrm{AH}$, Hisham $\mathrm{AN}$. Adrenal tuberculosis: The atypical presentations of eggshell-like calcifications. ANZ J Surg. 2009;79(6):488-9.

27. Gómez Rodríguez S, Pavón de Paz I, Torán Ranero CE, Modroño Móstoles N, Pérez Blanco C. Afecciones de ayer y hoy. Enfermedad de Addison de causa tuberculosa. Semer - Med Fam [Internet]. 2016;42(6):4168. Available from: http://linkinghub.elsevier.com/retrieve/pii/ S1138359315002750 